\title{
DISCONTINUOUS GALERKIN COMPUTATION OF GASEOUS MIXTURE COAXIAL JETS
}

\author{
N. Franchina ${ }^{1}$, M. Savini ${ }^{1}$, and F. Bassi ${ }^{1}$ \\ ${ }^{1}$ Università degli Studi di Bergamo, Dipartimento di Ingegneria e Scienze Applicate \\ viale Marconi, 5 Dalmine BG, Italy \\ e-mail: \{nicoletta.franchina,marco.savini,francesco.bassi\}@unibg.it
}

Keywords: Discontinuous Galerkin, high-order accurate discretization, multicomponent flow, pressure $L^{2}$ projection, preferential diffusion, coaxial jets

\begin{abstract}
A novel approach based upon Discontinuous Galerkin (DG) discretization, applied to the divergence form of the multicomponent Navier-Stokes equations, is here presented and used to compute non reactive turbulent axisymmetric gaseous jets. The original key feature is the use of $L^{2}$-projection form of the (perfect gas) equation of state. This choice mitigates problems typically encountered by the front-capturing schemes in computing multicomponent flow fields, i.e. spurious oscillations across material and contact surfaces where the mixture composition is changing. The solver makes also use of a shock-capturing technique based on artificial dissipation selectively added into the equations and tuned in connection with the magnitude of inviscid residuals of the equations and on suitable coefficients accounting for the variation of the unknown variables within and across grid elements. A simple limiting procedure is introduced in order to avoid the occurrence of unphysical gas properties due to negative and/or greater that one mass fractions values within the domain. The DG code based on the proposed novel technique for multicomponent flow computation is here employed to study the mixing mode and the preferential diffusion mechanism of a mixture jet of helium and carbon dioxide in a surrounding flow of air, both in laminar and turbulent flow regimes. Mass diffusion is modelled by means of Fick's first law and use is made of constant Prandtl and Schmidt numbers in Wilcox's (2008) $k-\omega$ model. Third-order accurate results are presented, discussed and compared with the available experimental data. They confirm the possible existence in coaxial jets of different periodic flow structures, greatly affecting mixing rates, and different species diffusive mass fluxes. The relative importance of both phenomena depends on the flow regime and its characteristics. The tests carried out give at the same time indications about the accuracy of the proposed method and its effectiveness in computing complex unsteady flow fields.
\end{abstract}




\section{INTRODUCTION}

The increased power and enhanced performance of the available computational resources achieved during these last years allowed a more widespread use of high-order accurate discretizations for the numerical prediction of complex flow fields of academic and industrial interest. The discontinuous Galerkin finite element method is one of the most promising numerical method due to the great geometrical flexibility, good scalability for high-performance computing and inherent high-order accuracy.

In the current work, the development of the DG code MIGALE [1, 2, 3, 4] for the computation of multicomponent flows, is presented. The solver is considered as one of our preliminary steps to deal with homogeneous (gas phase) combustion problems by means of a high-order CFD-DG tool and at present it neglects the modeling of chemical reactions.

The increasing importance of an accurate prediction of combustion processes, especially for the control of pollutant emissions, is nowadays indeed demanding further development of high-fidelity solvers, accurate enough to trace the evolution of chemical species down to ppm scale and, at the same time, robust and reliable enough to be applied to problems of industrial interest. The DG approach marries the ability to allow discontinuities in a natural way with its great accuracy granted even on general meshes, both important properties to compute the wrinkled flame fronts encountered in turbulent combustion, and indeed reactive DG codes have recently appeared (see e.g. [5, 6]).

The study of coaxial jets, with and without swirl, is of great importance in the assessment of the burner efficiency and it is an essential requirement to subsequently investigate the burner behaviour with partially premixed and non-premixed flames. In addition to this, the impact of coaxial jets fluid dynamics is crucial not only on the performances of rockets and combustion devices, but also in non-reactive components such as injectors and exhausts of turbofan engines. Studies on the behaviour of coaxial jets date back to the seventies with the pioneering experimental investigations presented in [7, 8, 9]; recent works [10, 11] confirm the existence of different flow configurations depending on the velocity and density ratios between inner and outer jets, as well as on geometrical details of the apparatus. The two main distinctive features are the "wake-like" and "jet-like" turbulent shear structures that, roughly speaking, develop respectively when the jets velocity ratio is near unity or far away from this value. Typically the flow field is turbulent and unsteady, with large scale fluctuations generated by Kelvin-Helmoltz instabilities at the shear interface between the jets, superimposed on the broad-band small scale turbulent background; thus the accurate simulation of gaseous coaxial jets represents indeed a challenging task for any multicomponent flow solver. Moreover, the growing interest in the use of syngas derived both from biomasses and coal, possibly added by methane in order to raise its enthalpy content (LHV), and/or in methane enrichment by hydrogen (to increase its flame speed and its lean stability limit) in turbogas burners, draws the attention toward the study of the effects induced by the faster mixing of one species (e.g. $\left.H_{2}\right)$, as compared with other species, on the whole process of combustion. As shown in [12], these effects are quite significant at low Reynolds numbers.

Numerical investigation on the mixing and diffusion phenomena in a coaxial jet of helium and carbon dioxide in air, at two different velocity ratios, is herein carried out using the multicomponent DG MIGALE code. The adopted DG solver is based on the "exact" Riemann solver [13] and on the BR2 scheme [1] for the computation of the interface convective and diffusive flux vector, respectively. It is well-known that the discretization of multicomponent flow fields has to cope with numerical oscillations that are not encountered with single component 
flows. Unphysical fluctuations may appear near contact and material interfaces, where the mixture composition changes, even with low-order accurate discretizations, depending on the sort of approximation adopted, see e.g. [14, 15]. Following the approach presented in [16] to mitigate spurious pressure oscillations at contact/material interface, the pressure is computed from the weak form of the equation of state for ideal gases. This can be viewed as a "regularization" of pressure that reduces its polynomial order of representation. Numerical oscillations that may also occur, due to flow field discontinuities, are treated by means of a shock-capturing scheme that explicitly adds artificial dissipation based on the inviscid residuals of the equations.

Two unsteady test cases have been computed, with jet-to-coflow velocity ratio $V$ ranging from 5 to 1 , and their results compared with experiments carried out at the Politecnico di Milano [17]. The computations allow a twofold check on the accuracy of the developed code, capable to investigate both the different mixing modes in coaxial jets and the concurrent mechanism of preferential diffusion of mass species.

In Section 2 we first describe the governing system of equations adopted for multicomponent gas mixture and the formulas employed to compute the mixture properties, then the DG approximation, the artificial dissipation and limiting procedure are presented. Section 3 reports the computed solutions along with a discussion on the jet structures and the mixing of chemical species. Final remarks are summarized in Section 4.

\section{NUMERICAL METHOD}

The numerical discretization presented in this section applies to the conservative form of the RANS equations. Their system for turbulent non reactive multicomponent flows is obtained through Favre averaging, neglecting the contributions due to the product of temperature and species mass fractions fluctuations. The system is written for both planar and axisymmetric 2D flows as follows

$$
\begin{aligned}
& \frac{\partial}{\partial t}(\rho r)+\frac{\partial}{\partial x}\left(\rho r \mathrm{v}_{x}\right)+\frac{\partial}{\partial r}\left(\rho r \mathrm{v}_{r}\right)=0, \\
& \frac{\partial}{\partial t}\left(\rho r \mathrm{v}_{x}\right)+\frac{\partial}{\partial x}\left[r\left(p+\rho \mathrm{v}_{x}^{2}\right)\right]+\frac{\partial}{\partial r}\left(\rho r \mathrm{v}_{x} \mathrm{v}_{r}\right)=\frac{\partial}{\partial x}\left(r \tau_{x x}\right)+\frac{\partial}{\partial r}\left(r \tau_{x r}\right) \\
& \frac{\partial}{\partial t}\left(\rho r \mathrm{v}_{r}\right)+\frac{\partial}{\partial x}\left(\rho r \mathrm{v}_{x} \mathrm{v}_{r}\right)+\frac{\partial}{\partial r}\left[r\left(p+\rho \mathrm{v}_{r}^{2}\right)\right]-\phi p=\frac{\partial}{\partial x}\left(r \tau_{x r}\right)+\frac{\partial}{\partial r}\left(r \tau_{r r}\right), \\
& \frac{\partial}{\partial t}\left(\rho r e_{t}\right)+\frac{\partial}{\partial x}\left(\rho r h_{t} \mathrm{v}_{x}\right)+\frac{\partial}{\partial r}\left(\rho r h_{t} \mathrm{v}_{r}\right)=A-r P+\beta^{\star} \rho r \bar{k} \mathrm{e}^{\widetilde{\omega}_{r}}+\frac{\partial}{\partial x}\left(r h_{i} J_{i, x}\right)+\frac{\partial}{\partial r}\left(r h_{i} J_{i, r}\right) \\
& \frac{\partial}{\partial t}(\rho r k)+\frac{\partial}{\partial x}\left(\rho r \mathrm{v}_{x} k\right)+\frac{\partial}{\partial r}\left(\rho r \mathrm{v}_{r} k\right)=\frac{\partial}{\partial x}\left(\bar{\mu}_{k} r \frac{\partial k}{\partial x}\right)+\frac{\partial}{\partial r}\left(\bar{\mu}_{k} r \frac{\partial k}{\partial r}\right)+r P-\beta^{\star} \rho r \bar{k} \mathrm{e}^{\widetilde{\omega}_{r}}, \\
& \frac{\partial}{\partial t}(\rho r \widetilde{\omega})+\frac{\partial}{\partial x}\left(\rho r \mathrm{v}_{x} \widetilde{\omega}\right)+\frac{\partial}{\partial r}\left(\rho r \mathrm{v}_{r} \widetilde{\omega}\right)=\frac{\partial}{\partial x}\left(\bar{\mu}_{\omega} r \frac{\partial \widetilde{\omega}}{\partial x}\right)+\frac{\partial}{\partial r}\left(\bar{\mu}_{\omega} r \frac{\partial \widetilde{\omega}}{\partial r}\right)+\frac{\alpha r \widetilde{\omega}}{\bar{k}} P-\beta \rho r \mathrm{e}^{\widetilde{\omega}_{r}},
\end{aligned}
$$

$$
\frac{\partial}{\partial t}\left(\rho r y_{i}\right)+\frac{\partial}{\partial x}\left(\rho r \mathrm{v}_{x} y_{i}\right)+\frac{\partial}{\partial r}\left(\rho r \mathrm{v}_{r} y_{i}\right)=\frac{\partial}{\partial x}\left(r J_{i, x}\right)+\frac{\partial}{\partial r}\left(r J_{i, r}\right), \quad i=1, \ldots, \mathrm{N}-1,
$$

where $\rho, p, \mathbf{v}=\left(\mathrm{v}_{x}, \mathrm{v}_{r}\right), e_{t}, h_{t}=e_{t}+p / \rho$, are the density, pressure, velocity, total internal energy and total enthalpy of the mean motion respectively, while $y_{i}$ is the mass fraction of the 
$i$-th species. The inclusion of the geometrical factor $r$ allows to use a unique implementation; $r$ is equal to the radius in the axisymmetric case and equal to one in the planar case, whilst the coefficient $\phi$ in Eq. (3) is equal to one and zero in the former and latter case, respectively.

Equations (1) and (7) express the conservation of the whole mass mixture and of $\mathrm{N}-1$ mass species, while Equations (2)-(4) express the momentum and energy conservation. As the total mass of the mixture is the sum of the mass of all species, the last (N-th) species conservation equation could be added to (7) in place of Eq. (1). Our choice, which is widely used in academic and commercial codes, ensures the global mass conservation, unaffected by chemical reactions and diffusive transport, and implicitly assumes that

$$
y_{\mathrm{N}}=1-\sum_{i=1}^{\mathrm{N}-1} y_{i}, \quad y_{i} \geq 0 \quad \forall i=1, \ldots, \mathrm{N}
$$

instead of computing the mixture density as $\rho=\sum_{i=1}^{\mathrm{N}} \rho_{i}$.

The term $A$ in the energy equation (4) summarizes the divergence of the work of laminar stresses and the divergence of the "effective" heat flux vector $\mathbf{q}_{h}^{e}$, defined as

$$
\mathbf{q}_{h}^{e}=-\left(k+\frac{\mu_{t} c_{p}}{P r_{t}}\right) \nabla T
$$

$P$ is the production term that models the energy exchange between mean and turbulent field (see [18] for their complete expressions).

The diffusive mass flux of the $i$-th species $J_{i}$ of Eqs. (4) and (7) is computed according to the Fick's first law

$$
J_{i}=-\rho D_{i, m}^{e} \nabla y_{i},
$$

where $D_{i, m}^{e}$ is the "effective" mass diffusion coefficient of the species $i$ in the mixture obtained as the sum of molecular and turbulent contributions,

$$
D_{i, m}^{e}=D_{i, m}+\mu_{t} /\left(\rho S c_{t}\right)
$$

and $S c_{t}$ is the turbulent Schmidt number introduced to model the product of species and velocity fluctuations through the classical gradient diffusion hypothesis. The $D_{i, m}$ coefficient is computed using a modified form of the Stefan's formula reported in [19], where $y_{i}$ has been replaced by $X_{i}$ (molar fraction of the $i$-th species) for self-consistency, i.e. at least when all $D_{i j}=D$, the formula guarantees that $D_{i m}=D$, and hence $\sum_{i=1}^{N} J_{i}=0$,

$$
D_{i, m}=\frac{1-X_{i}}{\sum_{j=1, j \neq i}^{\mathrm{N}} \frac{X_{j}}{D_{i j}}}
$$

The binary diffusion coefficients $D_{i j}$ are approximated as

$$
D_{i, j}=D_{(i, j)_{0}} \frac{\left(T / T_{0}\right)^{n}}{p / p_{0}},
$$

where $D_{(i, j)_{0}}$ is a reference diffusion coefficient at a reference state given by the pressure and temperature values $p_{0}$ and $T_{0}([20])$. Due to the constraint on the sum of mass diffusive fluxes, 
$J_{\mathrm{N}}=-\sum_{i=1}^{\mathrm{N}-1} J_{i}$, the mass diffusivity coefficient of the last species in the mixture $D_{\mathrm{N}, m}$ is implicitly defined as

$$
D_{\mathrm{N}, m}=\frac{\sum_{i=1}^{\mathrm{N}-1} D_{i, m} \nabla y_{i}}{\sum_{i=1}^{\mathrm{N}-1} \nabla y_{i}} .
$$

Note that, being working with non-reactive flows, the mass diffusion due to temperature gradients (Soret effect), as well as terms related to pressure gradients and body forces, have been neglected.

The diffusion flux $J_{i}$ gives rise to an energy flux $h_{i} J_{i}$ which, summed over all the species present in the mixture, provides the last terms in the RHS of Eq. (4), where the summation convention over repeated indices is adopted.

The turbulence model employed is the "high-Reynolds" version of Wilcox's $k-\omega$ turbulence model [21, 22]. Notice that the model adopted is not in its standard form and employs the modifications introduced in [2], i.e., in Equations (4)-(6) the turbulent kinetic energy $k$ is replaced by a limited value $\bar{k}$ to avoid the occurrence of negative values; besides the model uses $\widetilde{\omega}=\log \omega$ instead of $\omega$. Furthermore, "realizable" $\widetilde{\omega}_{r}$ (i.e. properly limited $\widetilde{\omega}$ ) values are used in the source term of Equation (6) and in the eddy viscosity computation to guarantee physical turbulent stress values. The standard RANS model employed makes use of the simple assumption of constant turbulent Prandtl number and of unique constant turbulent Schmidt number for each species. In this work they are fixed at 0.9 and 0.75 , respectively.

The dynamic viscosity $\mu$ and thermal conductivity $\lambda$ of the mixture are computed using the Wilke's formula [23] and the corrected expression given in [24] respectively, (see [25])

$$
\mu=\sum_{i=1}^{N} \frac{X_{i} \mu_{i}}{\sum_{j=1}^{N} X_{j} \phi_{i j}}, \quad \lambda=\sum_{i=1}^{N} \frac{X_{i} \lambda_{i}}{\sum_{j=1}^{N} X_{j} \phi_{i j}}
$$

with

$$
\phi_{i j}=\frac{\left\{1+\left(\frac{\mu_{i}}{\mu_{j}}\right)^{1 / 2}\left(\frac{R_{i}}{R_{j}}\right)^{1 / 4}\right\}^{2}}{\left\{8\left(1+\frac{R_{j}}{R_{i}}\right)\right\}^{1 / 2}} .
$$

The specific heat at constant pressure $c_{p, i}$, the dynamic viscosity $\mu_{i}$ and the thermal conductivity $\lambda_{i}$ of each species $i$ are defined as a function of the temperature through polynomial expansion up to the desired order of approximation. As in the present application pressure and temperature are nearly constant, all species thermofluidynamic properties are assumed constant.

In the hypothesis of perfect gas, the constitutive equation of state for the mixture reads $p=$ $\rho R T$, where $R$ is the mixture gas constant, $\sum_{i=1}^{\mathrm{N}} y_{i} R_{i}$. Herein the weak form of the EOS is considered and $p$ is defined in a weak sense via an integral relation:

$$
\int v p \mathrm{~d} V=\int v \rho R T \mathrm{~d} V
$$


where $v$ is every function in the chosen functional space, belonging to the Hilbert space of square integrable functions. The pressure degrees of freedom $p_{j}$ are computed in a straightforward way as

$$
p_{j}(t)=\int v_{j} \rho R T d V,
$$

due to the selected basis functions $v$, orthonormal in the physical reference frame.

\subsection{Discontinuous Galerkin Discretization}

The governing equations (1)-(7) can be written in compact form as

$$
\frac{\partial \mathbf{u}}{\partial t}+\nabla \cdot \mathbf{F}(\mathbf{u}, \nabla \mathbf{u})+\mathbf{s}_{t}(\mathbf{u}, \nabla \mathbf{u})=\mathbf{0}
$$

where $\mathbf{u} \in \mathbb{R}^{m}$ is the unknown solution vector of the $m$ conservative variables, $\mathbf{F} \in \mathbb{R}^{m} \otimes \mathbb{R}^{d}$ is the sum of inviscid and viscous flux functions (with $d$ indicating the number of dimensions) and $\mathbf{s}_{t} \in \mathbb{R}^{m}$ is the turbulent source term vector.

The discretization is defined over a computational mesh made of a set of non-overlapping elements $\mathcal{T}_{h}=\{K\}$. We thus consider the system (17) in its weak form over $\mathcal{T}_{h}$ and use a discontinuous Galerkin scheme based on the following space consisting of polynomial functions,

$$
V_{h} \stackrel{\text { def }}{=}\left[\mathbb{P}_{d}^{k}\left(\mathcal{T}_{h}\right)\right]^{m}
$$

where

$$
\mathbb{P}_{d}^{k}\left(\mathcal{T}_{h}\right) \stackrel{\text { def }}{=}\left\{v_{h} \in L^{2}(\Omega) \mid v_{h \mid K} \in \mathbb{P}_{d}^{k}(K), \forall K \in \mathcal{T}_{h}\right\}
$$

being $\mathbb{P}_{d}^{k}(K)$ the space of polynomials of degree at most $k$ defined on the element $K$. The DG discretization requires to compute $\mathbf{u}_{h} \in V_{h}$, such that:

$$
\begin{aligned}
\sum_{K \in \mathcal{T}_{h}} & \int_{K} v_{i} v_{j} \frac{\mathrm{d} U_{j}}{\mathrm{~d} t} r \mathrm{~d} \mathbf{x}-\sum_{K \in \mathcal{T}_{h}} \int_{K} \frac{\partial v_{i}}{\partial x_{n}} F_{j, n}\left(\mathbf{u}_{h}, \nabla_{h} \mathbf{u}_{h}+\mathbf{R}\left(\llbracket \mathbf{u}_{h} \rrbracket\right)\right) r \mathrm{~d} \mathbf{x} \\
& +\sum_{e \in \mathcal{F}} \int_{e} \llbracket v_{i} \rrbracket_{n} \widehat{F}_{j, n}\left(\mathbf{u}_{h}^{ \pm},\left(\nabla_{h} \mathbf{u}_{h}+\eta_{e} \mathbf{r}_{e} \llbracket \mathbf{u}_{h} \rrbracket\right)^{ \pm}\right) r \mathrm{~d} \sigma \\
& +\sum_{K \in \mathcal{T}_{h}} \int_{K} v_{i} s_{j}\left(\mathbf{u}_{h}, \nabla_{h} \mathbf{u}_{h}+\mathbf{R}\left(\llbracket \mathbf{u}_{h} \rrbracket\right)\right) r \mathrm{~d} \mathbf{x}=0,
\end{aligned}
$$

for each function $v_{i} \in V_{h}$, where $s_{j}=s_{t, j}+s_{a x, j}$ is the sum of turbulent and axisymmetric source terms. In Eq. (19) $\mathcal{F}$ defines the collection of all edges $e$ and the functions $\mathbf{R}$ and $\mathbf{r}_{e}:\left[L^{2}(e)\right]^{d} \rightarrow\left[\mathbb{P}_{d}^{k}\left(\mathcal{T}_{h}\right)\right]^{d}$ are the global and local lifting operators

$$
\begin{gathered}
\int_{\Omega} \mathbf{r}_{e}(\mathbf{v}) \cdot \boldsymbol{\tau}_{h} \mathrm{~d} \mathbf{x}=-\int_{e}\{\boldsymbol{\tau}\} \cdot \mathbf{v} \mathrm{d} \sigma, \quad \forall \boldsymbol{\tau}_{h} \in\left[\mathbb{P}_{d}^{k}\left(\mathcal{T}_{h}\right)\right]^{d} \\
\mathbf{R}(\mathbf{v})=\sum_{e \in \mathcal{F}} \mathbf{r}_{e}(\mathbf{v}),
\end{gathered}
$$

used by the BR2 scheme [1] to define a DG discretization of the viscous part of the governing equations. The jump $\llbracket \cdot \rrbracket$ and average $\{\cdot\}$ trace operators are defined as usual in the DG context to conveniently deal with discontinuities at elements interface as

$$
\llbracket \mathbf{u}_{h} \rrbracket \stackrel{\text { def }}{=} \mathbf{u}_{h}^{+} \mathbf{n}^{+}+\mathbf{u}_{h}^{-} \mathbf{n}^{-}, \quad\left\{v_{h}\right\} \stackrel{\text { def }}{=} \frac{v_{h}^{+}+v_{h}^{-}}{2},
$$


where the \pm superscripts indicate the trace of the numerical solution over a generic edge computed from the two adjacent elements $K^{+}$and $K^{-}$. The coefficient $\eta_{e}$ is a stability parameter depending on the number of elements faces; see [26] for a detailed analysis of the BR2 method.

Suitable numerical flux functions $\left(\widehat{\mathbf{F}}_{j}\right.$ in Eq. $(19)$ ) are used in place of the physical fluxes (convective and diffusive) to properly deal with the non-uniqueness of these vectors on the elements interfaces. The inviscid part of the flux is computed via "exact" Riemann solver [13] while the diffusive part as the average of the fluxes computed from neighboring elements.

The conservative formulation of the governing equations is used only for the purpose of fluxes computation, whilst the unknowns are given by the primitive variables vector

$$
\mathbf{q}=\left[\rho, T, v_{x}, v_{r}, k, \widetilde{\omega}, y_{1}, y_{2}, \ldots, y_{\mathrm{N}-1}\right]^{T} .
$$

The use of the primitive variables is computationally more efficient in the evaluation of the diffusive fluxes, where the gradient of $\mathbf{q}$ is required, since it is already available. Differently from monocomponent flow simulations, in multi-fluid flow computations we employ the density $\rho$ in place of the pressure $p$. In most reactive gas dynamic environments the pressure is in fact slowly varying or nearly homogeneous, whereas strong density gradients exist near flame fronts and thermal boundary layers.

The new multicomponent DG solver here presented closely follows the implementation of the monocomponent DG MIGALE code [1, 2, 3, 4]. A fully implicit discretization is employed with Jacobian matrices analytically computed to account in exact manner for the dependence of residuals on the values of variables and gradients, also including the treatment of lifting operators and boundary conditions.

\subsection{Time Integration}

The DG space discretization of the governing equations (19) is here written in the form of the following non-linear ODE system

$$
\mathbf{M} \frac{\mathrm{d} \mathbf{U}}{\mathrm{d} t}+\mathbf{R}(\mathbf{U})=\mathbf{0}
$$

where $\mathbf{U}$ is the vector of global unknown degrees of freedom, $\mathbf{M}$ the global block diagonal mass matrix and $\mathbf{R}$ the residuals vector. The time discretization of Eq. (23) is efficiently performed by means of a linearly implicit Rosenbrock-type Runge-Kutta schemes, following the implementation presented in [27], that can be written as:

$$
\begin{gathered}
\mathbf{U}^{n+1}=\mathbf{U}^{n}+\sum_{j=1}^{s} m_{j} \mathbf{W}_{j} \\
\left(\frac{\mathbf{I}}{\gamma \Delta t}+\widetilde{\mathbf{J}}\right)^{n} \mathbf{W}_{i}=-\widetilde{\mathbf{R}}\left(\mathbf{U}^{n}+\sum_{j=1}^{i-1} a_{i j} \mathbf{W}_{j}\right)+\sum_{j=1}^{i-1} \frac{c_{i j}}{\Delta t} \mathbf{W}_{j}, \quad i=1, \ldots, s,
\end{gathered}
$$

where

$$
\mathbf{J}=\frac{\partial \mathbf{R}}{\partial \mathbf{U}}, \quad \widetilde{\mathbf{R}}=\mathbf{M}^{-1} \mathbf{R}, \quad \widetilde{\mathbf{J}}=\frac{\partial \widetilde{\mathbf{R}}}{\partial \mathbf{U}}=\mathbf{M}^{-1}\left(\mathbf{J}-\frac{\partial \mathbf{M}}{\partial \mathbf{U}} \widetilde{\mathbf{R}}\right),
$$

and $c_{i j}, a_{i j}, m_{j}, \gamma$ are the scheme coefficients. The Eq. 25] is then replaced by the following efficient implementation that avoids the computation of $\mathbf{M}^{-1} \widetilde{\mathbf{J}}$,

$$
\left(\frac{\mathbf{M}}{\gamma \Delta t}+\mathbf{J}-\frac{\partial \mathbf{M}}{\partial \mathbf{U}} \widetilde{\mathbf{R}}\right)^{n} \mathbf{W}_{i}=-\mathbf{M}^{n}\left[\widetilde{\mathbf{R}}\left(\mathbf{W}^{n}+\sum_{j=1}^{i-1} a_{i j} \mathbf{W}_{j}\right)-\sum_{j=1}^{i-1} \frac{c_{i j}}{\Delta t} \mathbf{W}_{j}\right]
$$


for $i=1, \ldots, s$. For steady problems the time discretization is performed using the backward Euler scheme with the Jacobian matrix analytically computed to achieve quadratic convergence. For unsteady computations, accurate Runge-Kutta schemes up to fourth-order accuracy are adopted.

The linear system is finally solved using the restarted GMRES algorithm with 120 Krylov vectors, 240 iterations and a relative tolerance of $10^{-6}$. The code is parallel and employs the METIS package to handle grid partitioning and the PETSc library [28] for linear algebra.

\subsection{Shock-capturing technique}

High-order DG methods, like any linear (i.e. independent of the local solution) high-order method, suffer from numerical oscillations in the vicinity of flow field discontinuities. In multicomponent flow computations, spurious fluctuations appear near material fronts already with a first-order approximate conservative scheme. Thus, to obtain a crisp representation of discontinuities and preserve the accuracy within smooth flow field regions, it is necessary to adopt ad-hoc numerical techniques, such as shock capturing schemes suited to control oscillations.

In this work we employ the shock-capturing technique presented in [16], originally inspired by [29] which is based on the explicit introduction of artificial viscosity into the governing equations. The following element-wise artificial dissipation contribution is thereby added to the system (19):

$$
\int_{K} \varepsilon\left(\mathbf{u}_{h}^{ \pm}, \mathbf{u}_{h}\right) \frac{\partial v_{i}}{\partial x_{n}} \frac{\partial u_{j}}{\partial x_{n}} \mathrm{~d} \mathbf{x}
$$

The artificial viscosity coefficient $\varepsilon$ depends on two residual-based quantities: i) the difference between the numerical and physical inviscid fluxes and ii) the divergence of the inner convective fluxes,

$$
\varepsilon=C h_{K}^{2} \sum_{n=1}^{3}\left(\frac{\left|S_{n}\right|+\left|D_{n}\right|}{\widetilde{q}_{n}^{*}}\right) F_{s},
$$

where $C$ is a user-defined value, $h_{K}$ the characteristic dimension of the element $K$ defined in $2 \mathrm{D}$ as

$$
h_{K}=\frac{1}{\sqrt{\frac{1}{(\Delta x)^{2}}+\frac{1}{(\Delta r)^{2}}}},
$$

where $\Delta x$ and $\Delta r$ are the dimensions of the quadrilateral enclosing $K$, scaled in such a way that their product matches the area of $K$.

The viscosity is selectively introduced based on the behavior of the variables $\widetilde{\mathbf{q}}=\left(\rho, T, y_{M}\right)$, i.e. the density, the temperature and the mass fraction $y_{M}$ of the species showing the local greatest variation, chosen to tune the dissipation. Notice that in Eq. (29), the vector $\widetilde{\mathbf{q}}^{*}=$ $(\rho, T, 1)$ is used instead of $\widetilde{\mathbf{q}}$ to avoid break-up caused by too small values of $y_{M}$. The $S_{n}$ terms account for changes occurring at elements interface and based on the difference between the "numerical" and inner convective flux, herein denominated $s_{i}$. The $D_{n}$ terms account for any steep gradient in the flow field occurring within the elements and are based on the divergence of the convective inner flux, denoted by $d_{i}$,

$$
S_{n}\left(\mathbf{u}_{h}^{ \pm}, \mathbf{u}_{h}\right)=\sum_{i=1}^{M} \frac{\partial \widetilde{q_{h_{n}}}\left(\mathbf{u}_{h}\right)}{\partial \mathbf{u}_{h_{i}}} s_{i}\left(\mathbf{u}_{h}^{ \pm}\right), \quad D_{n}\left(\mathbf{u}_{h}\right)=\sum_{i=1}^{M} \frac{\partial \widetilde{q_{h_{n}}}\left(\mathbf{u}_{h}\right)}{\partial \mathbf{u}_{h_{i}}} d_{i}\left(\mathbf{u}_{h}\right)
$$


The artificial viscosity is then multiplied by a factor $F_{s}$ based on the maximum absolute value of the temperature, density and mass fraction scaled gradients, depending on polynomial order $k$ and mesh coarseness parameter $h_{K}$, given by

$$
F_{s}=\left(\left|\mathbf{d} \tilde{\mathbf{q}}_{\mathbf{j}}\right| \frac{h_{K}}{k}\right)^{k},
$$

with

$$
\left|\mathbf{d} \widetilde{\mathbf{q}}_{\mathbf{j}}\right|=\max _{j}\left(\frac{\left|\nabla_{h} \widetilde{q}_{j}\right|}{\widetilde{q}_{j}^{*}}\right), \quad j=1,2,3 .
$$

The user-defined coefficient $C$ has been determined through numerical experiments and in the present work it typically varies between 0.1 and 1 .

\subsection{Limiting on mixture properties}

During computations, out of bounds mass fraction values may appear in the solution flow field and this may lead to the attainment of unphysical gas properties. Different strategies have been considered by various authors to deal with oscillations arising in non monotonic approximation scheme, e.g. [30]. In this work, we do not constraint species degrees of freedom and thus negative or greater than one mass fraction values may appear. To keep this potential problem under control, we employed a simple limiting procedure that locally uses modified mass fraction values, satisfying Eq. (8), for the sole purpose of gas properties computation. The technique is presented in [16]; it first corrects (if necessary) each of the $\mathrm{N}$ species mass fraction to a minimum value and then divides each one by the sum of the new $\mathrm{N}$ mass fraction values.

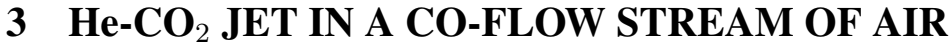

In this section third-order accurate $\left(\mathbb{P}^{2}\right)$ computations are discussed and compared with available experimental data [17].

Experiments were performed at ambient conditions in a configuration typical of non-premixed coaxial burners, consisting of an inner long pipe $(x / D=37.5)$ delivering a mixture of helium (11.8\% on a mass basis) and carbon dioxide, surrounded by much a shorter air annulus $\left(x / D_{m}=3.9\right)$. The closed chamber diameter is 5.6 times the annulus outer diameter, therefore confinement effects should not be relevant. The post thickness is $3.5 \mathrm{~mm}$. Other geometrical parameters of the apparatus are listed in Table 1

\begin{tabular}{c|cccc} 
& jet & air inner & air outer & chamber \\
\hline Diameter & 8 & 15 & 36 & 200 \\
Length & 300 & 80 & 80 & 300 \\
\hline
\end{tabular}

Table 1: Test rig dimensions [mm]

The considered test cases are summarized in Table 2, where the Reynolds numbers, based on bulk velocity, (hydraulic) diameter and kinematic viscosity, are reported alongside with density $(D)$, velocity $(V)$ and momentum flux $(M)$ inner-to-outer ratios.

Notice that in [17], due to a typing error, what is referred to as bulk velocity is actually the centerline velocity at the exit. The flow regime is incompressible, being the jet freestream Mach number $M a_{\text {jet }} \simeq 0.025$.

An unstructured grid consisting of 42722 elements of mixed triangular and quadrilateral type is employed to discretize half geometry. Figure 1(a) shows the computational domain (bottom 


\begin{tabular}{c|ccccc} 
Case & $R e_{\text {jet }}$ & $R e_{\text {air }}$ & $V$ & $D$ & $M$ \\
\hline 1 & 3140 & 2350 & 4.82 & 0.695 & 16.13 \\
2 & 3140 & 9480 & 1.19 & 0.695 & 1.01 \\
\hline
\end{tabular}

Table 2: Test case Reynolds number, velocity, density and momentum flux ratios

part of figure) and a close up of the mixing region (top part) near the merging of jet and co-flow. Note that the whole length of the tube has been discretized since it is not long enough to obtain a fully developed pipe flow.

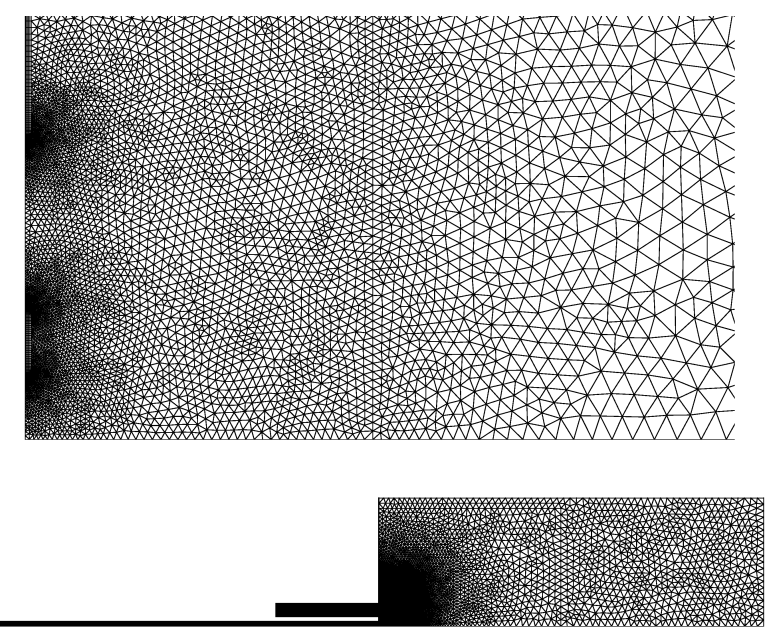

(a) grid



(b) time history

Figure 1: Computational mesh (left) and time histories of Case 1 - red and Case 2 -black (right)

The flow field is unsteady in both test cases, so computations were run in time-accurate mode until a periodic solution was finally set. To check the attainment of periodicity, fluidynamic quantities were continuously sampled at three points in the initial region of jets interaction $(x / D=1,0.5 \leq r / D \leq 0.95)$; an example of density time histories is shown in Figure 1(b).

In Case 1 both the inner and outer channel boundary layer Reynolds numbers are in the transitional range; even if they are well above the threshold for the development of turbulence in the subsequent jet free shear layer, flow field features may heavily depend on displacement and momentum thicknesses at the pipe exit. Therefore, for test Case 1 both laminar and turbulent computations were performed.

Figure 2 displays the Mach numbers and species mass fractions of the laminar solution at a generic instant of time, while Figure 3 shows the time-averaged periodic solution. At the jet exit, the shear layer (Kelvin-Helmholtz) instability generates a continuous detachment of vortices moving toward the axis, all counter-clockwise rotating, i.e. with positive vorticity. When they encounter their mirror image at the axis of symmetry, a local reinforcement is clearly visible prior to their slow downstream decay under the influence of viscosity. One single dominant frequency is present in the time spectrum, corresponding to a Strouhal number

$$
S t=\frac{\omega D}{V_{b u l k}} \simeq 0.49
$$




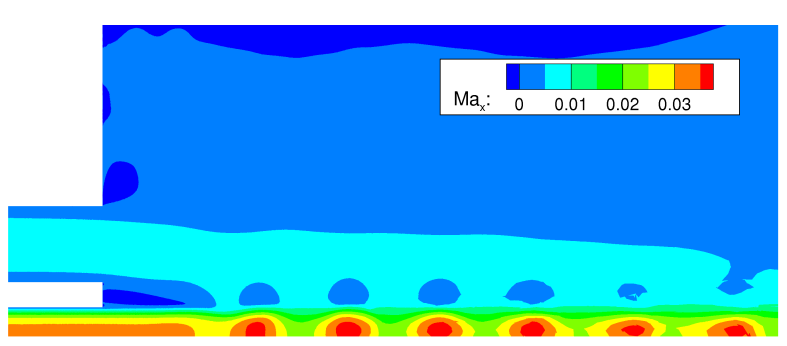

(a) $M a_{x}$

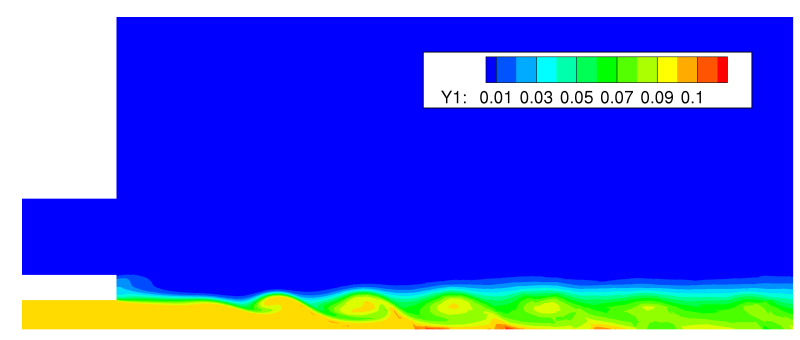

(c) $y_{H e}$



(b) $M a_{y}$



(d) $y_{\text {air }}$

Figure 2: Case 1, laminar: instantaneous contours



(a) $M a_{x}$

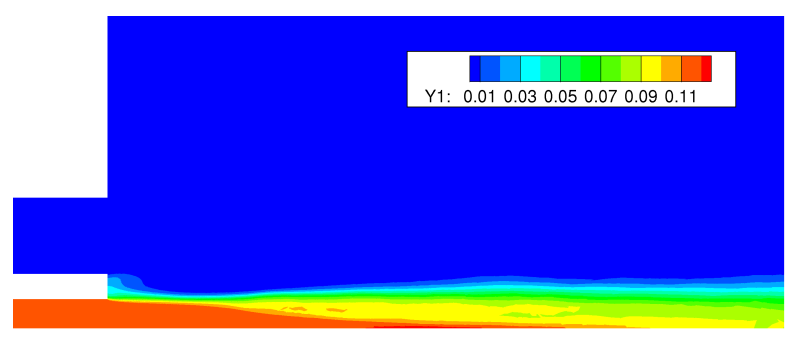

(b) $y_{\mathrm{He}}$

Figure 3: Case 1, laminar: time-averaged contours

In case it were a fully developed (Poiseuille) pipe flow, this would correspond to a Strouhal number based on momentum thickness of 0.021 , at the upper edge of the classical laminar range $(0.01-0.02)$.

In order to evaluate the mechanism of preferential diffusion, we compute a local diffusion parameter $\xi$ defined as follows,

$$
\xi=\frac{X_{\mathrm{CO}_{2}}}{X_{\mathrm{CO}_{2}}^{0}}-\frac{X_{\mathrm{He}}}{X_{\mathrm{He}}^{0}}
$$

where $X_{i}^{0}$ is the initial mole fraction of the species $i$. Positive values of this parameter can be interpreted as a local excess of carbon dioxide as compared with helium; the opposite is true for negative values.

Figure 4 shows the time-averaged numerical and experimental distributions of $\xi$ and axial non-dimensional velocity in the coaxial jets near field. As one can see, the laminar solution overestimates maxima and minima in both $\xi$ and velocity profiles; in particular, trace of the recirculation bubble due to the finite post thickness is still present at $x / D=0.75$, causing a zone of local backflow. We then turned to turbulent computations, using Wilcox's 2008 (high$\mathrm{Re})$ turbulence model. 


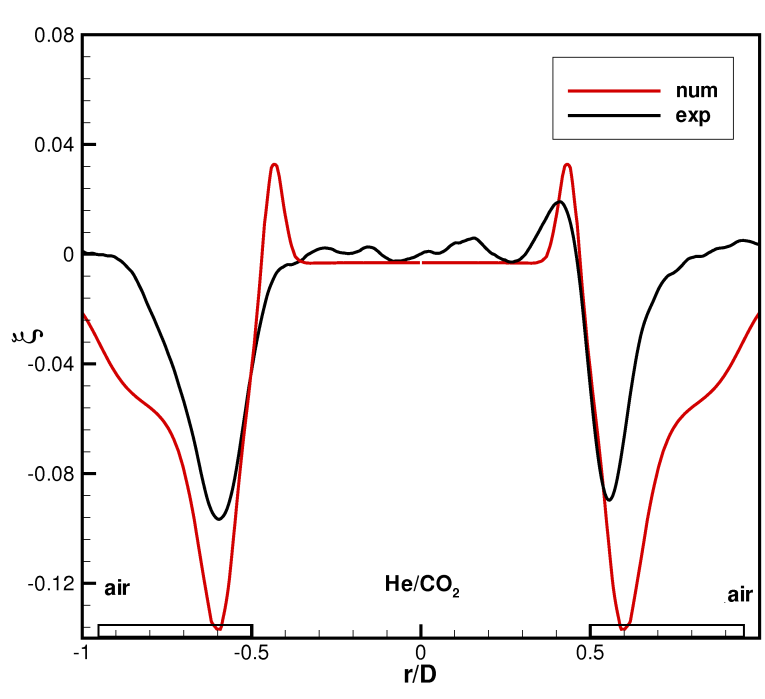

(a) $\xi$

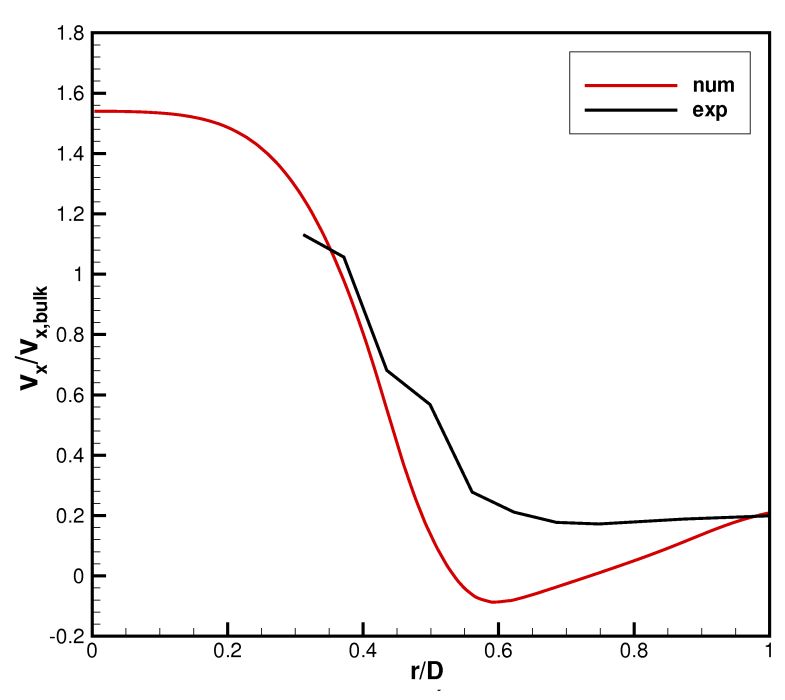

(b) velocity

Figure 4: Case 1, laminar: time-averaged $\xi$ coefficient and axial velocity at $x / D=0.75$

Figure 5 displays the Mach numbers, species mass fractions, vorticity and turbulence intensity of the turbulent solution at a generic instant of time, whilst Figure 6 shows the timeaveraged periodic solution. The vorticity is scaled with the pipe radius and the air ambient sound speed; in the turbulence intensity the turbulent kinetic energy is scaled with the square inlet air velocity. Unsteady coherent structures are still well evident, even if they are less pronounced and more smeared, owing to the diffusive action of turbulence. In the reported figures it can be noticed the different jet spreading rate, greater than in the laminar case, as should be expected. The axial distance travelled by perturbations before reaching the axis of symmetry is therefore shorter.

Figure 7 is, for the turbulent case, the companion of Figure 4. The results are now in a much better agreement with experimental data, confirming how the turbulence plays a key role in the mass and momentum mixing process. Maybe different results could have been obtained turning on turbulence in the simulation starting from the jet discharge axial point, but this is left as a future exercise. Test Case 2 was therefore only run under turbulent assumption.

Figure 8 shows a snapshot of the Mach numbers, species mass fractions, vorticity and turbulence intensity. This pictures are not at all alike their analogous obtained at greater velocity ratio. Vortices are leaving the air co-flow at the outer diameter as well and, moreover, the region enclosed between the two coaxial jets is now of the "wake-like" type with vortices of alternating sign visible close to the exit.

The coherent structures generated at the interface persist much longer downstream and the eventual setting of a self-similar solution is expected to occur farther away. The most important parameter governing what kind of configuration is likely to appear in the near field is indeed the momentum flux ratio $M$ and it is well established that a value of $M$ around unity leads to a deficit velocity profile, and possibly to an unsteady vortex street, typical of wake flows.

Furthermore, a much more complex flow field is apparent as a natural outcome of the jets strong mutual interaction. This is also verified in Figure 9 , where relevant steady spatial oscillations are noticeable. It is sensible to infer that removing the symmetry constraint and simulating the whole coaxial jet may result in a smoother average flow field, since perturbations are not forced to develop themselves simultaneously. 


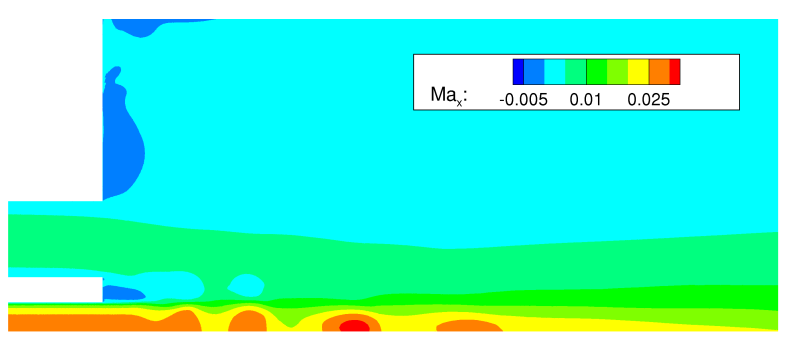

(a) $M a_{x}$

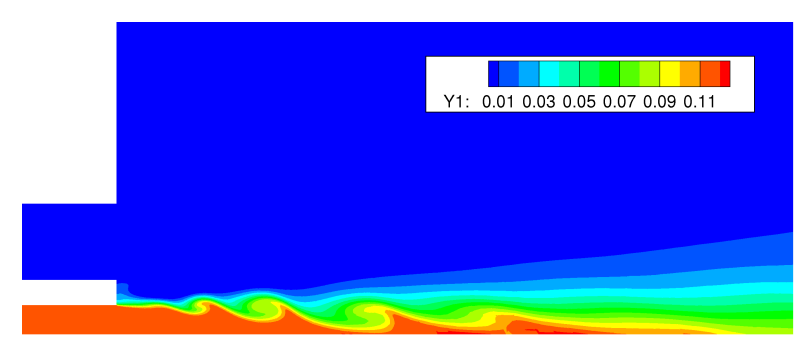

(c) $y_{\mathrm{He}}$

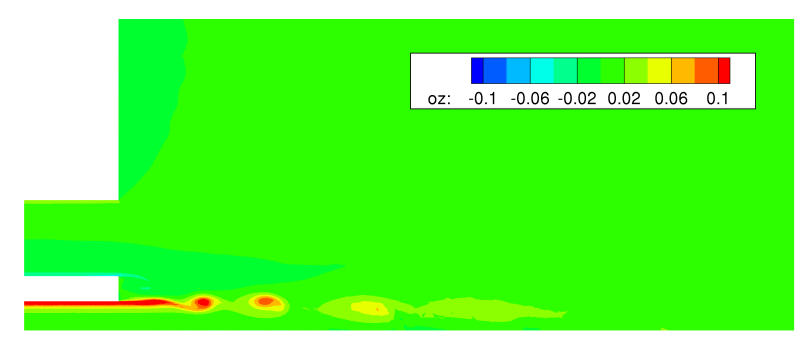

(e) vorticity

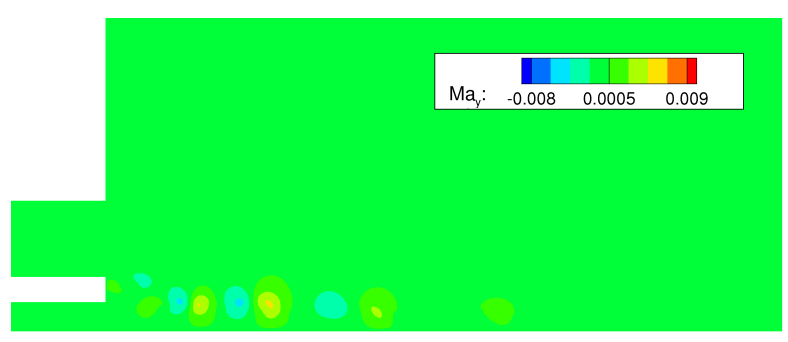

(b) $M a_{y}$

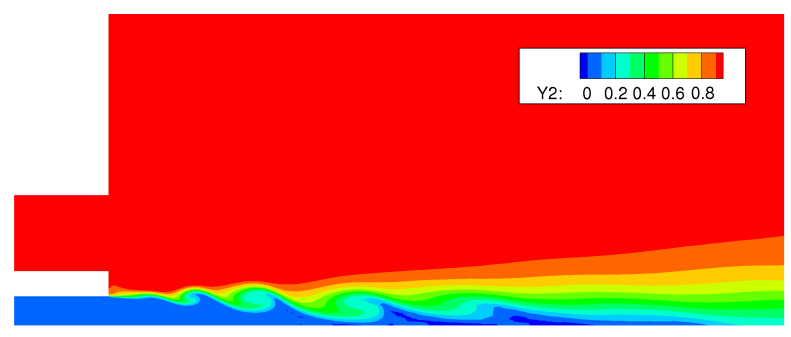

(d) $y_{\text {air }}$



(f) turbulence intensity

Figure 5: Case 1, turbulent: instantaneous contours

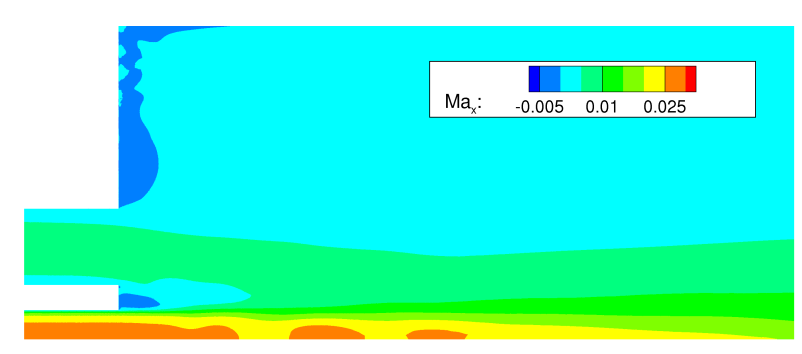

(a) $M a_{x}$

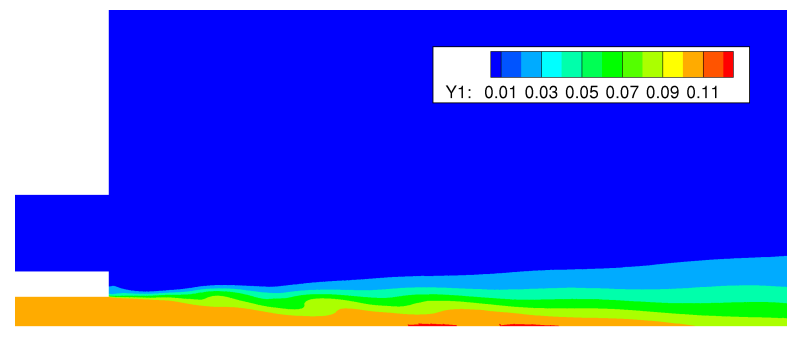

(b) $y_{\mathrm{He}}$

Figure 6: Case 1, turbulent: time-averaged contours 


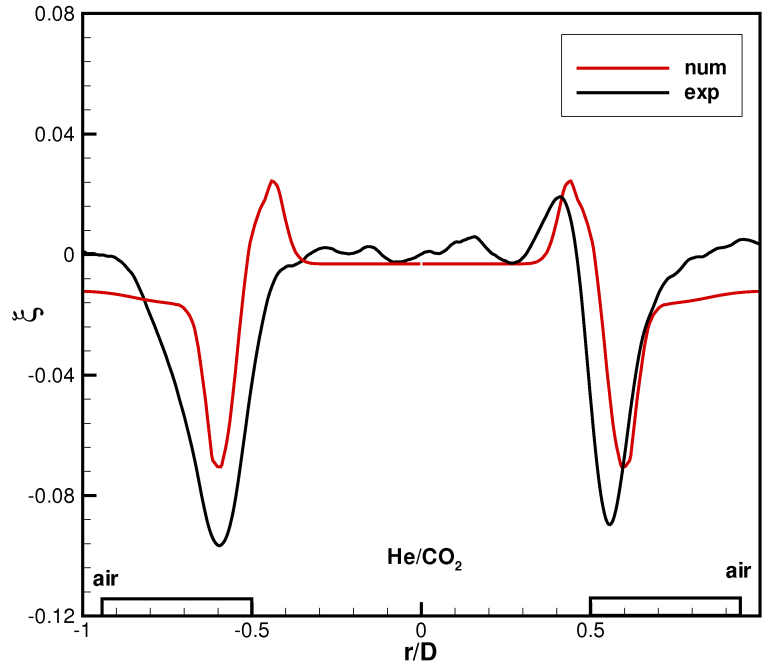

(a) $\xi$

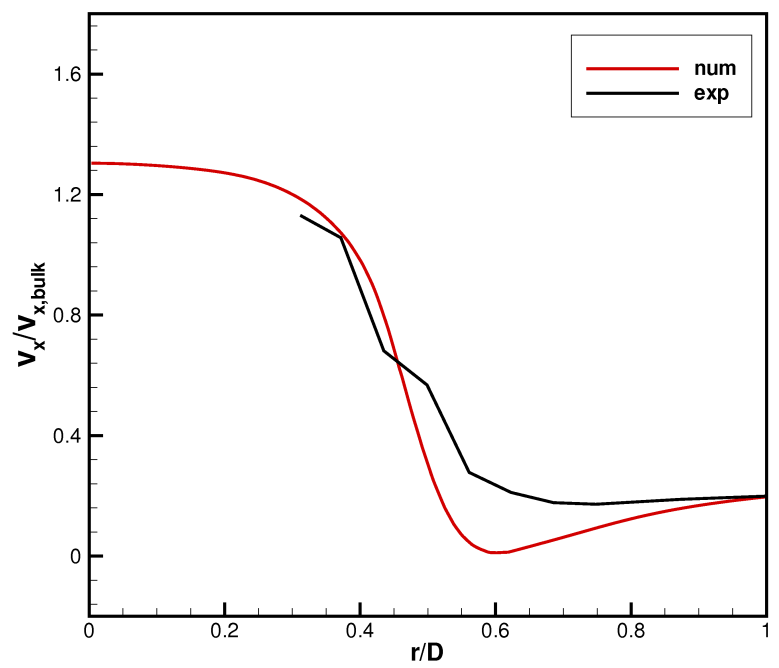

(b) velocity

Figure 7: Case 1, turbulent: time-averaged $\xi$ coefficient and axial velocity at $x / D=0.75$

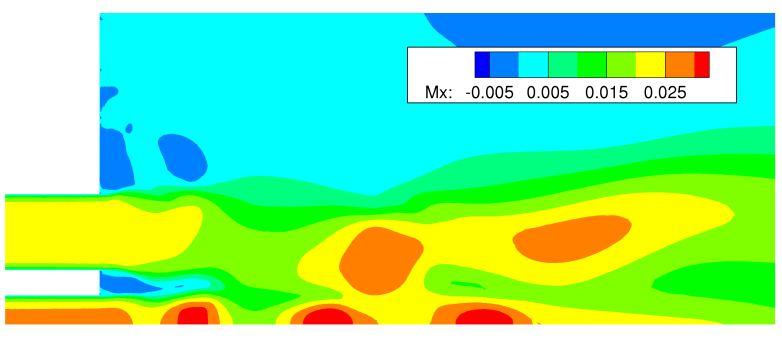

(a) $M a_{x}$

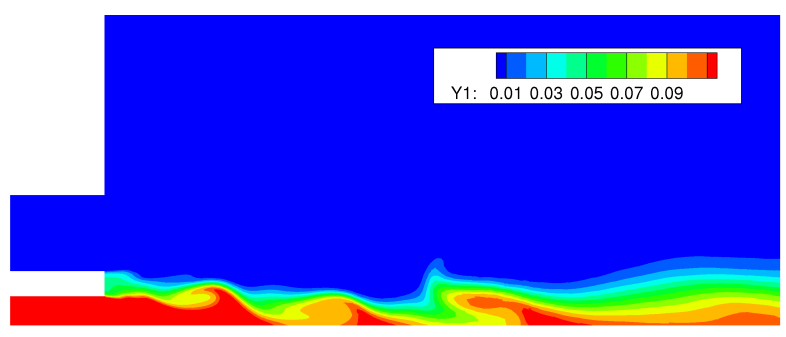

(c) $y_{\mathrm{He}}$

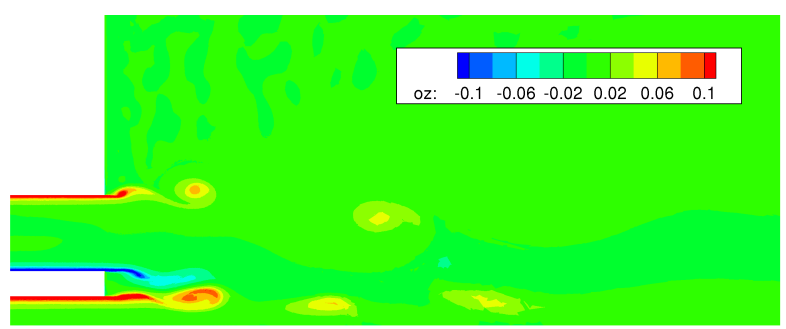

(e) vorticity

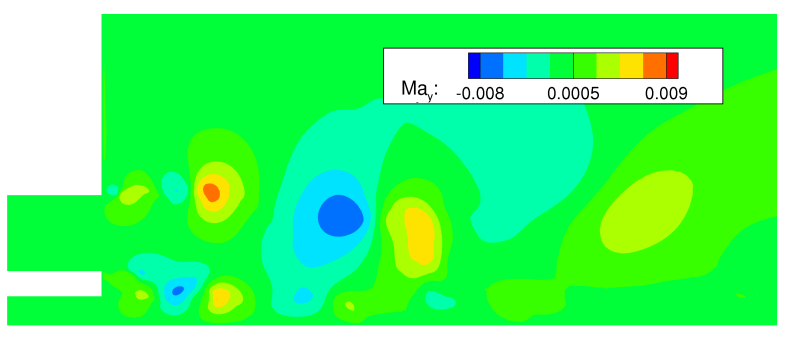

(b) $M a_{y}$



(d) $y_{a i r}$

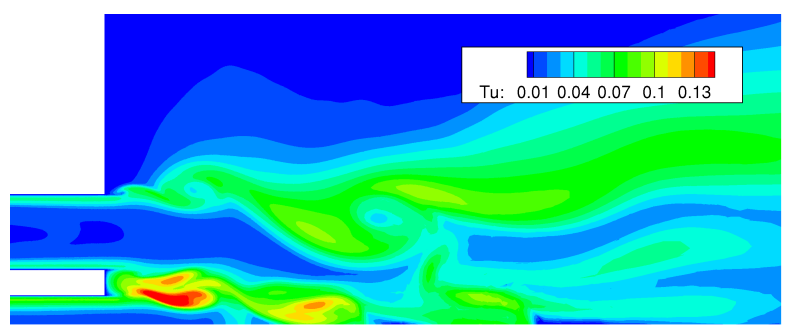

(f) turbulence intensity

Figure 8: Case 2: instantaneous contours 


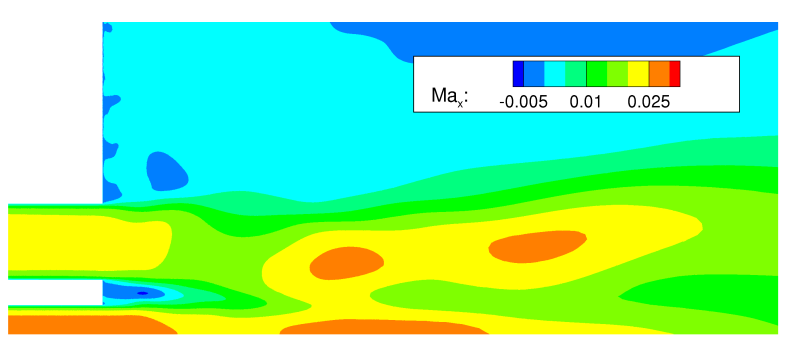

(a) $M a_{x}$

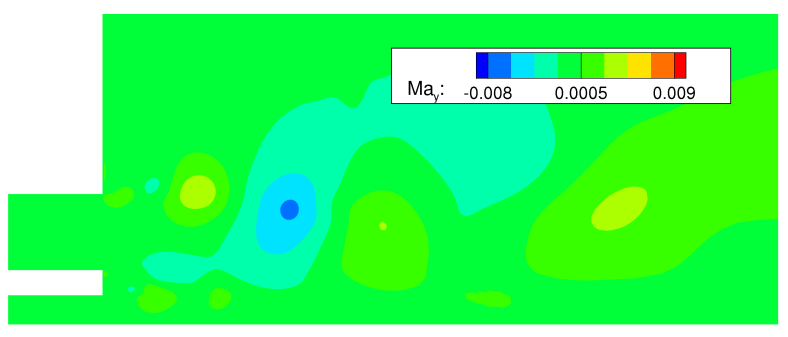

(c) $M a_{y}$

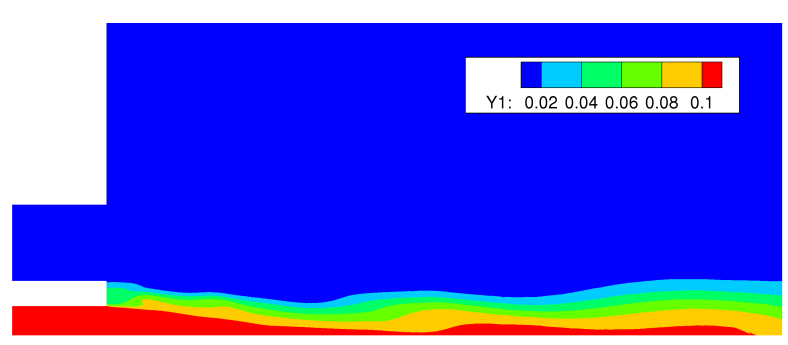

(b) $y_{\mathrm{He}}$

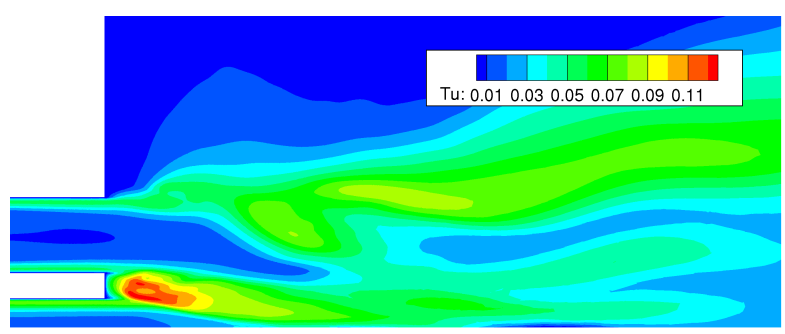

(d) turbulence intensity

Figure 9: Case 2: time averaged contours

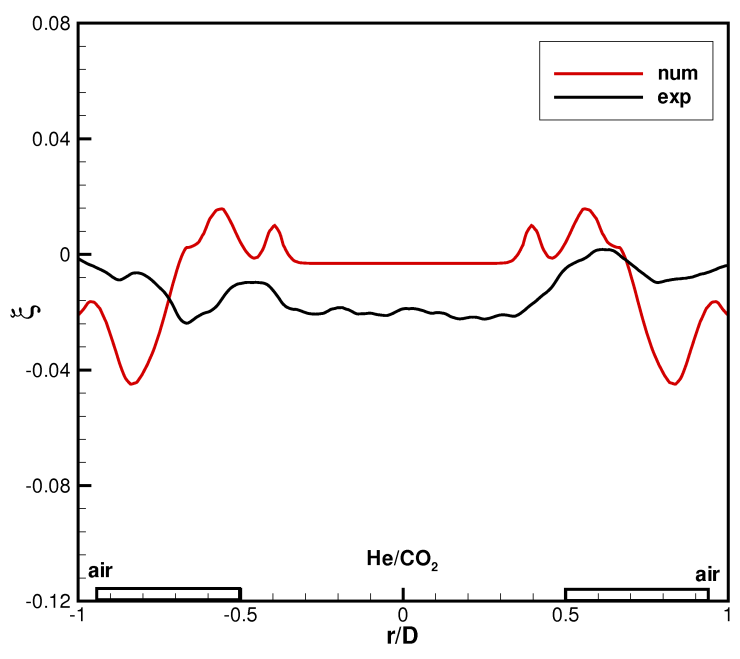

(a) $\xi$

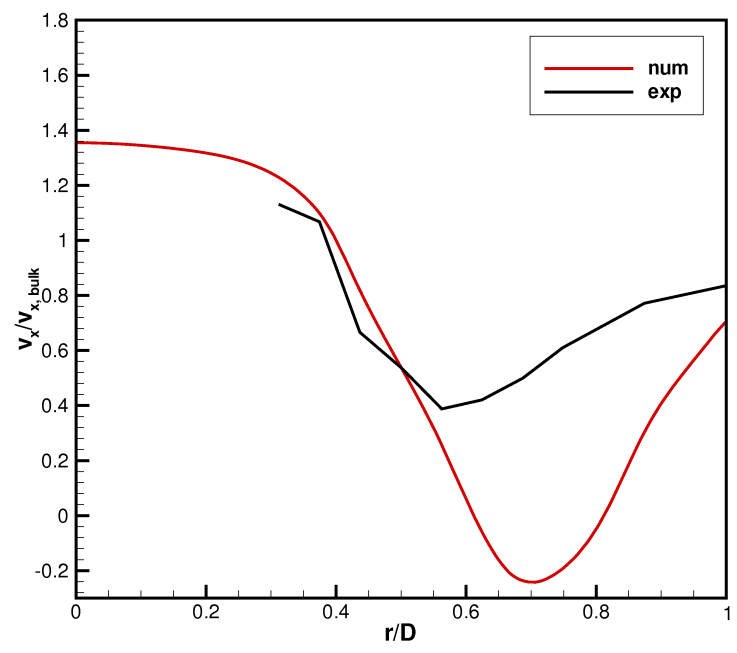

(b) velocity

Figure 10: Case 2: time-averaged $\xi$ coefficient and axial velocity at $x / D=0.75$

Time-averaged $\xi$ and non-dimensional $v_{x}$ graphs are reported in Figure 10 . The computed radial profile of helium and carbon dioxide mass fraction is satisfactory, keeping in mind the uncertainties inherent in the measurements (note the lack of symmetry and the nearly absence of positive values in the experimental data) and the fact that the local $\xi \mathrm{rms}$ is of the same order of its mean value; proof of this last statement can be found in Figure 11. On the other hand, there is an evident mismatch in the radial velocity profile. It seems due to the overestimation of the post recirculation zone in the numerical solution, similarly to what already manifest, even if to a lesser extent, in the Case 1 turbulent computation. The change of flow configuration from the "jet-like" to the "wake-like" type enlarged the rear separation bubble and shifted radially outward the point of minimum average velocity at $x / D=0.75$. 


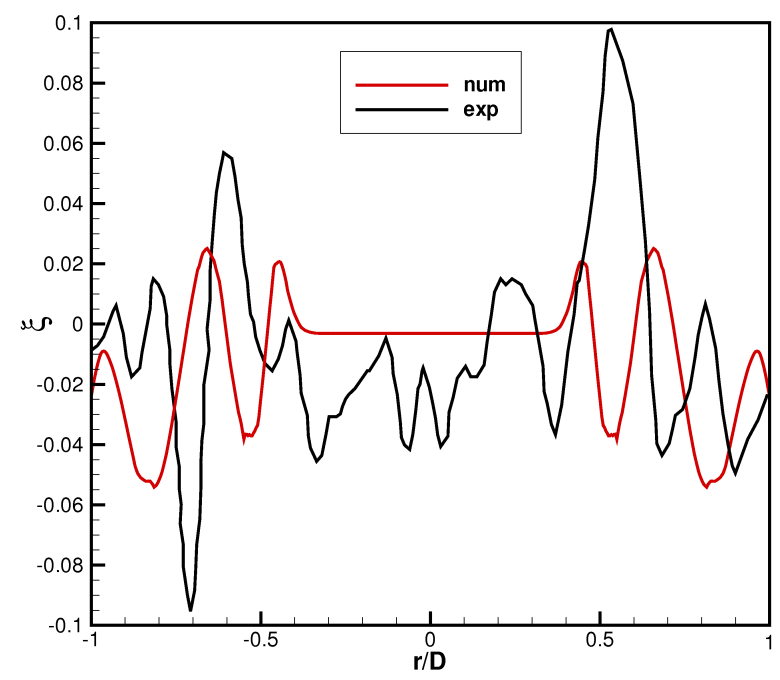

Figure 11: Case 2: instantaneous $\xi$ coefficient at $x / D=0.75$

\section{CONCLUSIONS}

A high-order accurate DG scheme suited to multicomponent flow computation has been presented and applied to investigate the complex mixing and diffusion mechanism occurring in coaxial jets. Oscillations typically encountered by numerical schemes applied to the conservative form of the multicomponent gas equations are herein kept under control by means of: i) the use of the weak form of the equation of state that allows to compute a more regular pressure field, ii) the addition of a shock-capturing term based upon local inviscid residuals and interface flux jumps, and iii) a limiting technique on mass fraction values, in order to avoid mixture properties out of admissible bounds.

The DG solver has been used to compute the flow of a mixture of helium and carbon dioxide discharging in a surrounding co-flow of air at two different Reynolds numbers and inner-toouter momentum flux ratios. Laminar and turbulent unsteady computations have been carried out to assess the capability of the code and to investigate the jet and co-flow mixing process taking place. The results obtained indicate in all cases the presence of a strongly unsteady flow field with the development of noteworthy coherent structures. Moreover, even at $R e$ as small as about $10^{3}$, the assumption of turbulent flow is necessary to obtain a reasonable agreement with measurements.

The preferential diffusion of helium with respect to carbon dioxide is evident (and well captured) only at the smaller $R e$ and higher velocity ratio, when also the computed time-averaged velocity field in the near region is close to the experimental one. In this case the mixing process resembles the one, well known, experienced by a jet emanating in a quiescent ambient, whereas a complete different picture exists when jet and co-flow possess similar velocity (more correctly, similar momentum flux). In this latter case one observes a "wake-like" mixing mode, characterized by a more complex interaction and a much long-lasting presence of vortices.

Finally, it is worth to mention that in the former case (jet) the unsteadiness is focused on a single time scale and the unsteady vorticity field is positive definite, while in the latter case (wake) more frequencies are present altogether with an unsteady vorticity field showing both signs. These and other interesting topics are currently under investigation and will be the subject of a forthcoming paper. 


\section{REFERENCES}

[1] F. Bassi, S. Rebay, G. Mariotti, S. Pedinotti, and M. Savini. A high-order accurate discontinuous finite element method for inviscid and viscous turbomachinery flows. In R. Decuypere and G. Dibelius, editors, 2nd European Conference on Turbomachinery Fluid Dynamics and Thermodynamics, pages 99-108, Antwerpen, Belgium, March 5-7 1997.

[2] F. Bassi, A. Crivellini, S. Rebay, and M. Savini. Discontinuous Galerkin solution of the Reynolds-averaged Navier-Stokes and $k$ - $\omega$ turbulence model equations. Comput. \& Fluids, 34:507-540, 2005.

[3] F. Bassi, A. Colombo, N. Franchina, A. Ghidoni, and S. Rebay. Robust and efficient implementation of very high-order discontinuous Galerkin methods in CFD. Notes on Fluid Mechanics, 113:287-299, 2010.

[4] F. Bassi, L. Botti, A. Colombo, A. Crivellini, C. De Bartolo, N. Franchina, A. Ghidoni, and S. Rebay. Time Integration in the Discontinuous Galerkin code MIGALE - Steady Problems. In Norbert Kroll, Charles Hirsch, Francesco Bassi, Craig Johnston, and Koen Hillewaert, editors, IDIHOM: Industrialization of High-Order Methods - A Top-Down Approach, volume 128 of Notes on Numerical Fluid Mechanics and Multidisciplinary Design, pages 179-204. Springer International Publishing, 2015.

[5] G. Billet, J. Ryan, and M. Borrel. A Runge Kutta Discontinuous Galerkin approach to solve reactive flows on conforming hybrid grids: the parabolic and source operators. Computers \& Fluids, 95:98-115, 2014.

[6] Y. Lv and M. Ihme. Discontinuous Galerkin method for multicomponent chemically reacting flows and combustion. J. Comput. Phys., 270:105-137, 2014.

[7] F. H. Champagne and I.J. Wygnanski. An experimental investigation of coaxial turbulent jets. J. Heat and mass transfer, 14:1445-1464, 1971.

[8] A. Antonia and R. W. Bilger. An experimental investigation of an axisymmetric jet in a co-flowing air stream. J. Fluid Mech., 61:805-822, 1973.

[9] N.W.M. Ko and A.S.H. Kwan. The initial region of coaxial jets. J. Fluid Mech., 73:305332, 1976.

[10] G. Buresti, P. Petagna, and A. Talamelli. Experimental investigation on the turbulent nearfield of coaxial jets. Exp. Thermal and Fluid Science, 17:18-36, 1998.

[11] S. A. Schumaker. An experimental investigation of reacting and nonreacting coaxial jet mixing in a laboratory rocket engine. PhD thesis, University of Michigan, 2009.

[12] F. Cozzi and A. Coghe. Behavior of hydrogen-enriched non-premixed swirled natural gas flames. Int. Journal of Hydrogen Energy, 31:669-677, 2006.

[13] J. J. Gottlieb and C. P. T. Groth. Assessment of Riemann solvers for unsteady onedimensional inviscid flows of perfect gases. J. Comput. Phys., 78:437-458, 1988.

[14] R. Abgrall. How to prevent pressure oscillations in multicomponent flow calculations: a quasi conservative approach. Rapporte de recherche, INRIA, 2372, Octobre 1994. 
[15] E. Johnsen and F. Ham. Preventing numerical errors generated by interface-capturing schemes in compressible multi-material flows. J. Comput. Phys., pages 5705-5717, 2012.

[16] N. Franchina, M. Savini, and F. Bassi. Multicomponent gas flow computations by a discontinuous Galerkin scheme using $L^{2}$-projection of perfect gas EOS. J. Comput. Phys., 2015, 10.1016/j.jcp.2016.03.059.

[17] F. Cozzi, A. Olivani, L. Caratti, and A. Coghe. Investigation of fine structure of turbulent and molecular diffusion in coaxial jets of $\mathrm{He} / \mathrm{CO}_{2}$ in air by LDA and Rayleigh scattering. Exp. Thermal and Fluid Science, 34:316-322, 2010.

[18] F. Bassi, F. Cecchi, N. Franchina, S. Rebay, and M. Savini. High-order discontinuous Galerkin computation of axisymmetric transonic flows in safety valves. Comput. \& Fluids, 49:203-213, 2011.

[19] J. Warnatz, U. Maas, and R.W. Robert. Combustion: Physical and Chemical Fundamentals, Modeling and Simulation. Springer, 2006.

[20] N. B. Vargaftik and Y.K. Vinogradov. Handbook of physical properties of liquid and gases: pure substances and mixtures. Begel House, 1996.

[21] D. C. Wilcox. Turbulence Modelling for CFD. DCW industries Inc., La Cañada, CA 91011, USA, 2006.

[22] D. C. Wilcox. Formulation of the k- $\omega$ turbulence model revisited. AIAA Journal, 44:28232838, 2008.

[23] C.R. Wilke. A viscosity equation for gas mixture. J. Chem. Phys., 18:517-519, 1950.

[24] E.A. Mason and S.C. Saxena. Physics of fluids. J. Fluids Eng., pages 361-369, 1958.

[25] B. R. Byron, W.E. Stewart, and E. N. Lightfoot. Transport phenomena. John Wiley \& Sons, Inc., 2002.

[26] D. N. Arnold, F. Brezzi, B. Cockburn, and L. D. Marini. Unified analysis of discontinuous Galerkin methods for elliptic problems. SIAM J. Numer. Anal., 39(5):1749-1779, 2002.

[27] F. Bassi, L. Botti, A. Colombo, A. Crivellini, A. Ghidoni, and F. Massa. Linearly implicit Rosenbrock-type Runge-Kutta schemes applied to the Discontinuous Galerkin solution of compressible and incompressible unsteady flows. Comput. \& Fluids, 118:305-320, 2015.

[28] S. Balay, S. Abhyankar, M. F. Adams, J. Brown, P. Brune, K. Buschelman, L. Dalcin, V. Eijkhout, W. D. Gropp, D. Kaushik, M. G. Knepley, L. Curfman McInnes, K. Rupp, B.F. Smith, S. Zampini, and H. Zhang. PETSc Web page. http://www.mcs.anl. gov/petsc, 2014.

[29] F. Bassi, L. Botti, A. Colombo, A. Crivellini, N. Franchina, A. Ghidoni, and S. Rebay. Very high-order discontinuous Galerkin computations of transonic turbulent flows on aeronautical configurations. Notes on Fluid Mechanics, 113:25-38, 2010.

[30] L. Krivodonova. Limiters for high-order discontinuous Galerkin methods. J. Comput. Phys., 226:879-896, 2007. 